\title{
Una experiencia de aula basada en los juegos de magia como herramienta pedagógica en Educación Primaria
}

A Classroom Experience based on Magic Cames as a Pedagogical Tool in Primary Education

\author{
IGNACIO GONZÁLEZ-PUELLES DE ANTONIO
}

Escuela Universitaria CEU de Magisterio de Vigo

$M^{a}$ SANDRA FRAGUEIRO BARREIRO

Escuela Universitaria CEU de Magisterio de Vigo

Recibido: 27/09/2017

Aceptado: 15/03/2018

\section{RESUMEN}

En este trabajo se presentan los resultados obtenidos tras la implementación de una propuesta innovadora centrada en el ilusionismo en un aula de Educación Primaria. Dicha experiencia surge con el fin de desarrollar numerosas competencias clave, tratando de potenciar un cambio metodológico y transformar los procesos de enseñanza-aprendizaje. Se exponen una fundamentación teórica, el diseño y puesta en práctica en un centro escolar y finalmente, la evaluación para concluir con una propuesta de mejora del mismo.

\section{ABSTRACT}

This document deals with the results obtained after the implementation of an innovative proposal. It is focused on illusionism in a Primary Education classroom. This experience arises in order to develop many competences, trying to promote a methodological change and transform the teaching-learning processes. Also the theoretical fundamentation will be exposed. The project design and the conclusions are also included ending up with an improvement proposal. riencia de aula basada en los juegos de magia como herramienta pedagó-

\section{KEYWORDS}

Illusionist, Innovative Education, Magic, Projects. 


\section{INTRODUCCIÓN}

La propuesta de intervención de este documento se basa en la incorporación del ilusionismo como recurso didáctico en el aula. Tras leer los trabajos e investigaciones que hicieron otros maestros y maestras sobre el uso del ilusionismo como herramienta metodológica en sus clases, surge esta propuesta innovadora de hacer un juego de manos para desarrollar numerosas competencias clave y habilidades, tanto motrices como cognitivas. Así, nuestro objetivo se centrará en implementar una experiencia de aula en un centro escolar utilizando el ilusionismo para potenciar un cambio metodológico y transformar los procesos de enseñanza-aprendizaje.

El presente trabajo está estructurado en tres partes principales: en primer lugar, se recogerá el estado de la cuestión centrado en la "magia educativa", así como una recopilación de las ventajas que esta metodología ofrece apoyándose en sus precursores. En segundo lugar, se detallará la propuesta de intervención: la metodología, las actividades centradas en los juegos de magia usados explicando los conocimientos que se buscaban potenciar, los recursos utilizados y la evaluación del proyecto en Educación Primaria. Para concluir, se expondrá una reflexión, tanto de lo que se ha aprendido y descubierto como de todas las mejoras a realizar.

\section{ESTADO DE LA CUESTIÓN}

En este trabajo el término "magia" está alejado de la connotación mística y sobrenatural con que es definido en la Real Academia Española (RAE): "arte o ciencia oculta con que se pretende producir, valiéndose de ciertos actos o palabras, o con la intervención de seres imaginables, resultados contrarios a las leyes naturales" o "encanto, hechizo o atractivo de alguien o algo". "Magia" guarda más relación con el concepto de prestidigitación que es "arte o habilidad de hacer juegos de manos y trucos para la distracción del público" (RAE, 2016).

La magia como entretenimiento tiene un origen desconocido. El primer documento que se ha encontrado sobre este tema es el papiro de Westcar datado hace más de cinco mil años en Egipto y descubierto en 1825. En este se relatan las proezas de un mago llamado Dedi. En una de ellas le cortó la cabeza a un ganso y después la volvió a unir como si nada hubiese pasado (Ludueña, 2015).

En el siglo XVI, Reginal Scott hace referencia al ilusionismo en su libro titulado "The discovery of witchcraft" ("El descubrimiento de la brujería”), publicado en 1584, en el que explica una gran cantidad de juegos de magia para alejar a los ilusionistas del rol de "brujo". A finales de este siglo, el maestro Gonin funda una dinastía de magos.

Durante el siglo XVII, las intervenciones de los magos aumentaron. La mayoría eran mercaderes ambulantes que aprovechaban sus números para vender objetos. En esta época se representó la primera obra de ilusionismo teatral y se escribieron los primeros tratados de magia.

El ilusionismo sigue desarrollándose a lo largo del tiempo hasta llegar a la mitad del siglo XIX, donde aparecen dos figuras que lo elevan hasta la calidad de arte y lo alejan aún más del rol de "brujo": Robert Houdin en Francia y Johannes Nepomuk Hofninser en Viena. Robert Houdin fue tan importante haciendo magia en el teatro que llegó a cambiar el estereotipo de mago que estaba concebido anteriormente por el que existe hoy en día, es decir, por el de mago con frac y chistera. Hofninser promovió tanto la magia con cartas, que hoy en día se siguen haciendo sus juegos.

El siglo XX es considerado por muchos como el siglo de oro de la magia gracias a magos como Max Malini, Kellar, Blackstone en el escenario, el escapista Houdini, o Dunninger y Anemman en el arte del mentalismo. 
En esta época se desarrolla la teoría sobre los mecanismos de la magia con magos como Dai Vernon y su búsqueda de la naturalidad, la teoría del control de la atención de Slydini, sin olvidar las investigaciones de la “Escuela Mágica de Madrid” con Ascanio, Tamariz, Carroll, etc. (Tamariz, 1991).

Hoy en día la magia se sigue desarrollando y fomentando, apareciendo publicaciones en congresos y en bibliografía especializada; surgen asociaciones de magos a nivel mundial y se incrementa su difusión en televisión con programas de Dough Henning en Estados Unidos, Juan Tamariz en España y Paul Daniels en Reino Unido, por citar algunos. Posteriormente, estos programas televisivos han sido sustituidos por un nuevo estilo de magia televisiva difundida por magos como Criss Angel, David Blaine o Dynamo, entre otros.

Tras este breve repaso en la historia de la magia, se tratará a continuación la rama de la magia que será utilizada en este documento, la magia infantil y educativa.

Ruiz (2013) denomina "magia educativa" a la forma de aplicar los juegos de manos con fines educativos. Kaye (2007), considerado el rey de la magia infantil en Nueva York, defiende en su libro Serio de remate: cómo hacer magia para niños que el siglo XXI es una época idónea para ser mago infantil. A pesar de ello, todavía es escasa la bibliografía publicada sobre este tema, apareciendo solamente algún artículo en revistas especializadas en Educación (Suárez, 2010; Francisco, Martínez y Sánchez, 2013), entrevistas a profesores que la aplican en el aula o información de cursos especializados en este recurso didáctico (García, 2011; Gomez y Camañero, 2009). Algunos de los libros hallados sobre el tema son: Educando con magia de Ruiz (2013), Ernesto, el aprendiz de matemago de Santoja (2003) y Matemagia de Blasco (2016).

El campo donde se ha hallado más documentación sobre esta unión de la magia con la enseñanza ha sido el de las matemáticas, gracias a juegos del área denominada por los magos "Matemagia”. Estos juegos basan su secreto en utilizar uno o varios principios matemáticos a la vez, por lo que sirven a los profesores para explicar diferentes operaciones y teoremas o para ponerlos en práctica de una manera más lúdica. Otra área donde se ha trabajado es dentro de la "Ciencia recreativa", aplicando experimentos lúdicos que pueden ser presentados como juegos de magia y usados para explicar diferentes principios (Almau, 2013).

A pesar de la escasez bibliográfica, existe en España una gran cantidad de maestros que están usando la magia en sus aulas, tales como Jordí Martin, Álvaro Conde, María Teresa Suárez Vacca, Sergio González Linares o Alejandro Hernández Nebra, entre otros. Algunos de estos profesores imparten cursos sobre el tema en los centros de profesorado y recursos de sus respectivas comunidades autónomas. Además existen proyectos centrados exclusivamente en esta vertiente de la magia como, por ejemplo, el proyecto educativo realizado en Castilla la Mancha y Madrid (Fernández y Serrano, 2015) para mejorar la comprensión matemática a través de la Matemagia que consiguió mejorar el nivel del alumnado o el trabajo de Juan Sebastián Barrero, quien recibió el premio Francisco Giner de los Ríos con su proyecto Matemagia en 2014 (Montesillos, 2014).

Fuera de España, en el área de matemáticas son varios los profesionales que aplican la magia como recurso didáctico en el aula. Koirala (2005) recoge un proyecto que utiliza un juego de magia para introducir a los alumnos en las funciones de primer grado. Nishiyama (2012) busca introducir la geometría a los alumnos usando juegos de magia basados en principios geométricos. Otros autores trabajan la probabilidad de una manera más lúdica y dinámica (Lane, 2010; Lopez, 2014; McShea, Vogel y Yarnevich, 2005).

Spencer (2001) trabaja con magia para ayudar a alumnos con dificultades de aprendizaje consiguiendo mejorar su capacidad para relacionarse con sus compañeros y mejorar su autoestima. 
Para completar esta investigación sobre la magia educativa se han usado como textos adicionales libros en los que se explica el secreto de diferentes trucos de magia tales como Gran curso de Magia y prestidigitación (De Vecchi, 2009) y Magia para niños (Presto, 1999) para poder adaptar algunos de los juegos en el contexto educativo.

Existe una serie de ventajas a la hora de usar la magia en el aula, tanto para el profesor como para el alumno. Una de las ventajas más importantes de la magia educativa, que influye en el proceso de enseñanza-aprendizaje es la relación recíproca que se genera, es decir, utilizarla para explicar ciertos contenidos que no se pueden observar directamente, tales como fenómenos de la naturaleza o leyes de la Física (Ruiz, 2013; Almau, 2013; González, 2011). Por ejemplo, se puede hacer que un coche de juguete tele-dirigido circule no solo por la pared, sino también por el techo. Después se les puede explicar a los alumnos que esto es causado por el efecto Venturi, que relaciona la velocidad y la presión; si se incrementa la corriente de aire que pasa por debajo del coche tele-dirigido, la presión se reducirá y si esta presión es menor que la atmosférica, hace que el coche se adhiera a la pared (Almau, 2013).

Otra ventaja radica en que la magia puede utilizarse para interiorizar conceptos a través de los denominados "ganchos mentales", es decir, el carácter lúdico de la magia hace que los contenidos se asimilen mejor. Además, si durante el juego ocurre algo sorprendente, los alumnos recordarán el contexto donde este se produjo, haciendo que los contenidos que se pretenden explicar se recuerden mejor (Martín, 2011). En la competencia matemática se aprecia esta capacidad de comprensión, como han demostrado los diferentes proyectos educativos que se han realizado en España, tales como el de Rafael Fernández Cezar y Francisco Javier Lahiguerra Serrano, aplicado en diversos colegios de las comunidades de Castilla la Mancha y Madrid (Fernández y Serrano, 2015). Esto es debido a que muchos de los secretos de los juegos de magia se basan en principios matemáticos (Navas, 2012). Estos juegos se pueden usar para que el alumno desarrolle la actividad matemática de una manera más recreativa, consiguiendo así una actitud más positiva hacia esta materia (Ruiz, 2013; Almau, 2013; Conde, 2013), por ejemplo, algunos juegos como "El piano" o "El cuadrado mágico" (Gardner, 1992). En el primero, los alumnos trabajan la diferencia entre los números pares e impares, mientras que en el segundo trabajan las sumas de varios sumandos y la propiedad conmutativa de la suma.

Una tercera ventaja es que se puede reforzar a través de la magia la competencia lingüística del alumnado, tanto a nivel escrito como oral. Para la escrita, Ruiz (2013) propone potenciarla a través de un libro redactado por los alumnos con las explicaciones de los juegos de magia que aprenden a lo largo del curso. Estas explicaciones deben figurar detalladas de forma que otra persona pueda hacer el juego leyendo exclusivamente las instrucciones. La capacidad oral se ve desarrollada cuando el alumno es el que realiza el juego de magia, pues debe hablar en un tono de voz elevado y vocalizando para que los espectadores puedan entender el juego de magia. Además, debido a que se debe transmitir una serie de emociones al espectador al realizar el juego, se potencia la inteligencia emocional (Conde, 2013). Esta se define como "una parte de la inteligencia social que incluye la capacidad de controlar nuestras emociones y las de los demás, discernir entre ellas y usar dicha información para guiar nuestro pensamiento y comportamiento" (Salovey, Mayer, Goldman, Turvey y Palfai, 1995). Además, cuando es el alumno el que realiza los juegos de manos, desarrolla otros instrumentos personales como seguridad, autoestima, concentración y las relaciones interpersonales (Conde, 2013). Estas últimas se ven aumentadas porque durante el juego existe una comunicación activa entre los espectadores y el mago. Además, tal y como dice Alejandro Hernández Nebra en una entrevista realizada por Almau (2013), "la magia es un idioma universal. Como hay muchos chavales que no están acostumbrados a recibir el reconocimiento de los demás, con la magia lo pueden recibir, es inclusiva y ayuda a la convivencia de la diversidad" (p. 40). 
Además de las ventajas anteriormente mencionadas, Conde (2013) argumenta que hacer magia ejercita la memoria para recordar las charlas y/o presentación del juego y la memoria muscular, pues el cuerpo debe retener los pasos de los juegos de manera que sean automáticos, dejando así espacio para la presentación. El hecho de que el alumno ensaye y realice un juego de magia también desarrolla la psicomotricidad fina y gruesa. La motricidad fina se ve reforzada, por ejemplo, cuando este rompe un papel, hace un nudo en una cuerda u oculta en su mano un objeto que acaba de escamotear. Mientras que la motricidad gruesa se potencia cuando este se mueve por el escenario o cuando tiene que realizar movimientos determinados así desarrolla la creatividad pues el alumno debe crear una presentación. Por otra parte, existen otras dos formas de desarrollar la creatividad con la magia: o bien haciendo un juego, teniendo ellos que explicar cómo lo han realizado o mediante la resolución directa de problemas que se les plantee (Conde, 2013).

Para los profesores una de las ventajas de los juegos de magia es su gran versatilidad. Cada tutor puede adaptarlos a lo que precise en cada momento, según el alumnado, etapa y materia. En la asignatura de educación artística (plástica) se pueden crear diferentes objetos usados en magia como, por ejemplo, la "caja de espejos" (Presto, 1999). Se muestra un ejemplo en la Figura 1. Esta usa un espejo azogado a cuarenta y cinco grados para dar la sensación de que está aparentemente vacía cuando no es así, por lo que se puede usar para hacer diferentes apariciones (Ruiz, 2013; Almau, 2013; Martín, 2011).

\section{Figura 1}

Ejemplo de una caja de espejos.

http://www.magicaplanet.com/images/prod/prod_Sc18.jpg)

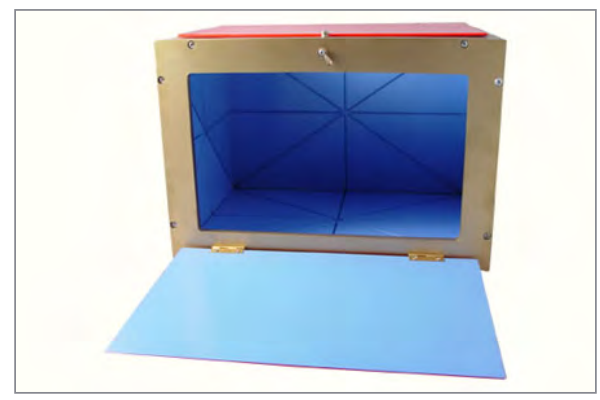

El uso de la magia en el aula mejora la motivación y el interés del alumnado, para la gran mayoría de autores. Esto es debido al componente lúdico que la magia presenta, haciendo que "se diviertan y se motiven tanto alumnos como adultos”, tal y como afirma González (2011). Además, si se realiza un juego de magia al inicio de la unidad didáctica se puede captar mejor la atención del alumno, haciendo que el aprendizaje sea más divertido y, en consecuencia, despertar el interés de este sobre el tema. Navaz (citado en Etcheverry, 2000) defiende que al utilizar el ilusionismo dentro del aula, se puede conseguir centrar la atención de los alumnos y alumnas, y una vez alcanzada esta concentración despertarán su curiosidad ante lo que el profesor vaya a explicar. Por lo tanto, es una herramienta pedagógica y didáctica que suma al proceso de enseñanza-aprendizaje la intriga, la curiosidad, el asombro y la motivación creando una "atmósfera mágica" que resultará una "explicación mágica" para unos alumnos motivados, atentos y con inquietudes por el gusto por aprender; y también se habrá salvado el obstáculo de la distracción y logrado un aprendizaje significativo. 
Muchos autores coinciden en que la prestidigitación genera una gran motivación y potencia el interés en los alumnos (Lovitt y Clarke, 1988; Koirala y Goodwin, 2000), intensifica la sensibilidad sensorial y fomenta la creatividad (Frith y Walker,1983), desarrolla el pensamiento crítico y favorece las habilidades para la resolución de problemas (McCormack, 1985).

Con la magia el profesor puede también controlar el comportamiento del alumnado a modo de "chantaje mágico". Para ello se les explica que, si su comportamiento es el adecuado, recibirán como premio el juego de magia. Según Ruiz (2013), existen dos formas para avisar que recibirán el premio: dibujando tres chisteras que se van tachando cada vez que el alumnado no se porte bien, reduciendo así el comportamiento negativo (este sistema fue usado en el proyecto denominándose "sistema de tres strikes"), o bien con otro método denominado por el autor como "un conejo en la chistera". Este método tiene un funcionamiento similar al anterior en cuanto se dibujan tres chisteras en la pizarra de la clase, difiriendo en que se les explica a los alumnos que dentro de la chistera vive "Manolito Rabbit". Si el conejo escucha ruido se quedará dentro de la chistera pero, si la clase se mantiene en silencio, el profesor dibuja las orejas del conejo saliendo de una de las chisteras. Si siguen portándose bien, dibuja la cabeza completa del conejo saliendo de la segunda chistera y, al final, si continúa el buen comportamiento, lo dibuja saliendo de la chistera con una varita mágica, símbolo de que habrá un juego de magia al final. En la Figura 2 se muestra la secuencia de dibujos puesta en práctica.

\section{Figura 2}

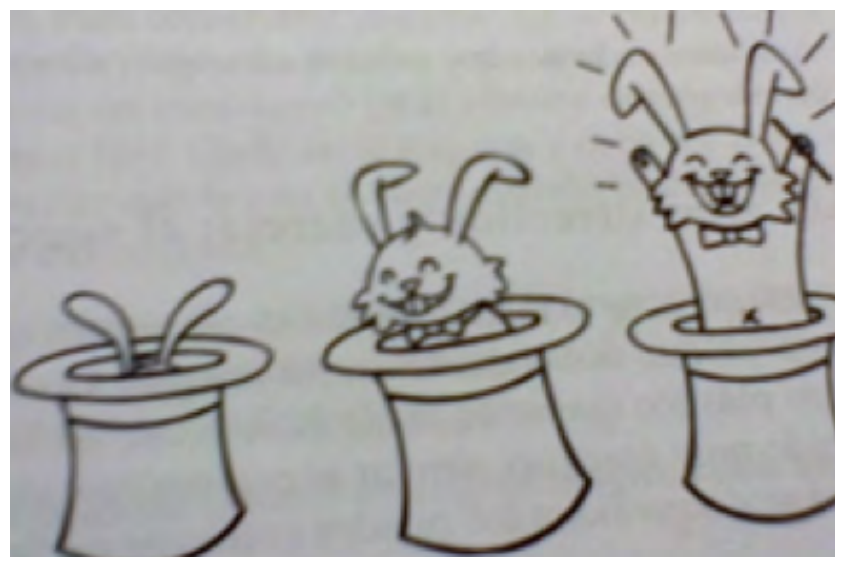

Para finalizar, cabe destacar que Ruiz (2013) también menciona otra serie de ventajas de la magia educativa tales como: la capacidad de llamar la atención del alumnado, ser un elemento mediador de conflictos entre los alumnos y ayudar a controlar un alumno conflictivo. 


\section{PROPUESTA DE INTERVENCIÓN}

Una vez planteada la fundamentación teórica sobre la que se sustenta nuestro trabajo, se diseña y se lleva a la práctica la propuesta de intervención basada en la magia educativa en un colegio de la ciudad de Vigo, concretamente en las aulas de $2^{\circ}$ y $6^{\circ}$ de Educación Primaria.

El objetivo principal que se plantea con esta experiencia de aula radica en potenciar y despertar en los discentes la motivación, el interés por participar y aprender en determinadas áreas del conocimiento a través del ilusionismo, lo que repercutirá en la mejora del comportamiento al crear un clima agradable en el aula. De esta forma se potencia que desarrollen ciertas competencias promoviendo un aprendizaje activo.

En cuanto a la metodología que inspira la implementación de las actividades centradas en la magia de este trabajo, lo primero que se ha tenido en cuenta a la hora de realizar estos juegos de magia es fomentar el carácter lúdico de las actividades de enseñanza-aprendizaje con el fin de conseguir la necesaria motivación hacia el aprendizaje, tal y como se indica en Ley Orgánica 8/2013 para la mejora educativa (LOMCE).

Aunque dichas actividades deberán ser motivadoras y gratificantes, no se debe perder de vista que estas deberán estar fundamentalmente dirigidas al logro de los objetivos y competencias clave, comunicativas, inclusivas, activas y participativas. Además, han de responder a sus intereses reales y situarse en su propio contexto vital.

En segundo lugar, con esta experiencia se busca generar aprendizajes tanto válidos como eficaces y desarrollar diferentes actividades mentales de los alumnos tales como la observación, la relación entre las ideas, conceptos y vivencias con la realidad pues son fuentes de progreso en el aprendizaje y en el desarrollo intelectual de los niños.

En tercer lugar, se ha buscado trabajar y desarrollar el mayor número de competencias clave posibles tales como la comunicación lingüística, la matemática, la científica y tecnológica, las sociales y cívicas, el sentido de la iniciativa y espíritu emprendedor y la conciencia y expresiones culturales.

Para controlar el comportamiento de los alumnos se ha usado el recurso de los tres strikes. Este recurso se basa en una manera de avisar al alumnado de su comportamiento y las consecuencias que este tendrá. En caso de que haya un comportamiento negativo se les avisa con un cartel en el que pone "Strike uno" y se pega en la pizarra tradicional. En caso de que se llegue al "Strike tres", los jueves no se hará la magia y será día de lectura normal. Este recurso está basado en las ideas que presenta Ruiz (2013) para controlar el comportamiento del alumnado en su libro Educando con magia.

Para atraer la atención del alumnado se cuenta con una serie de opciones, la primera es que la actividad se hacía dentro del horario lectivo. A la hora de empezar a realizar la actividad sonaba el timbre que avisa del fin de la clase, además de esto mientras los alumnos recogían los objetos el profesor se levantaba de su sitio, se colocaba en la pizarra enfrente de ellos y esperaba en silencio a que se sentasen y se callasen. Justo antes de empezar preguntaba en voz alta si querían que empezase.

Al realizar el proyecto, se usan diferentes estrategias metodológicas para conseguir que la misma actividad (en este caso un juego de magia) sea variada para su aprendizaje. Dependiendo del juego, la interacción con el alumnado cambia, en algunos casos se asemejaba al modelo de la clase magistral, en la que los alumnos solo observaban sin participar. En otros casos la participación del alumnado era indirecta, ya sea comprobando los elementos que se van a usar para hacer magia, respondiendo las preguntas planteadas por las magias o realizando el gesto mágico en momentos determinados. Finalmente, en algunos de estos juegos hay una participación directa del alumnado: no solo sacando a uno como voluntario, sino también siendo ellos los que realicen 
los juegos de magia. Durante la realización del trabajo hubo dos casos de este tipo: en uno, el alumno era guiado por el mago, y en otro, el propio alumno realizó un espectáculo de magia.

Tras el juego de magia se les planteaba un enigma que debían resolver. Estos enigmas buscaban desarrollar su creatividad, y se usaba un modelo de interrogatorio. Se les planteaba el enigma a los alumnos, y estos debían plantear su posible solución. En este caso siempre se les pedía que la justificasen y se les planteaba contraargumentos; de esta forma el alumno desarrollaba el razonamiento. En muchos casos se les decía la solución al día siguiente o incluso no se decía, pues el objetivo de estos enigmas se conseguía cuando los alumnos planteaban la solución.

En contadas ocasiones, se realizó magia sin estar en el tiempo de magia. Esto es debido a usar la magia como un "recurso polivalente", tal y como indica Ruiz (2013). Por ejemplo, tras volver a clase de una excursión se improvisó un show de magia para ocupar ese tiempo de una manera más divertida y además mejorar la comunicación con el alumnado. Otros casos se producían cuando impartía una de las asignaturas: se producía un efecto de magia que solo los alumnos que estaban atendiendo pudieron ver, con esto se conseguía mejorar la atención de la clase a la hora de impartir la asignatura.

\section{ACTIVIDADES}

A continuación, se detallan las actividades realizadas con el fin de desarrollar las competencias clave y de potenciar un cambio metodológico en los procesos de enseñanza-aprendizaje.

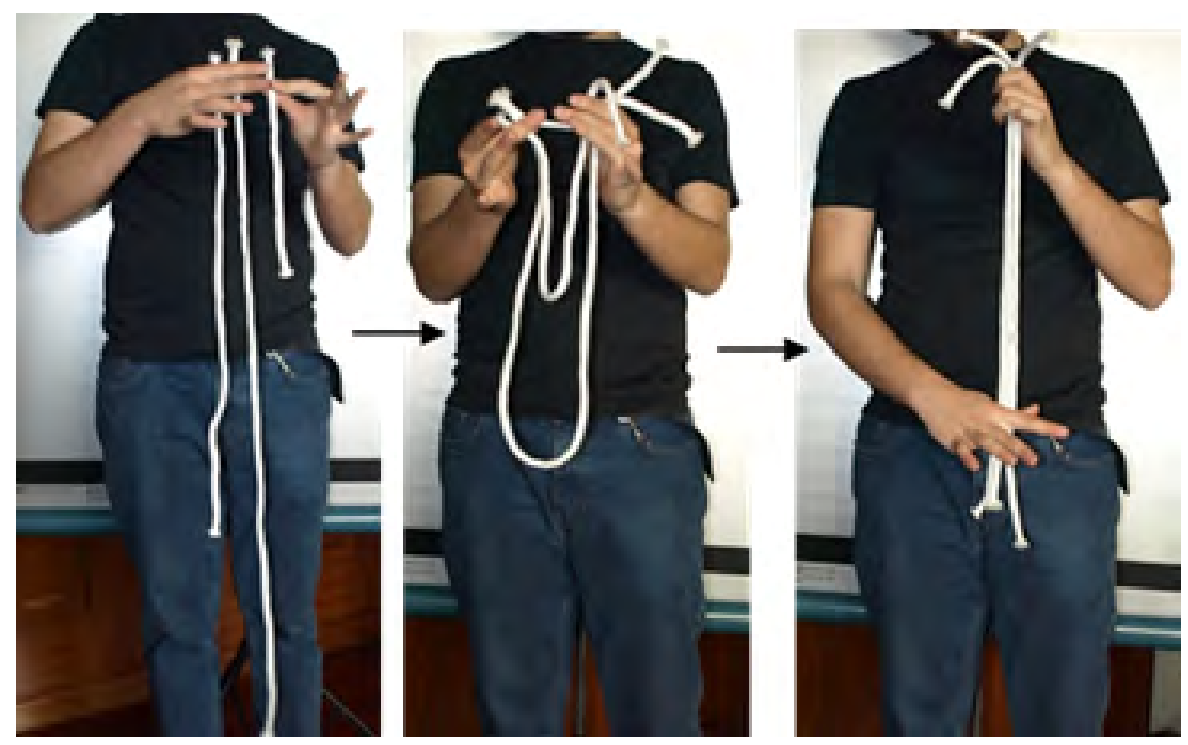




\subsection{Las cuerdas mágicas (Presto, 1999)}

Contenidos: medidas de longitud.

Competencias: competencia en comunicación lingüística, competencia matemática y competencias básicas en ciencia y tecnología.

Efecto: Se dejan tres cuerdas a diversos alumnos y se les pide que las midan usando sus manos. Cuando terminan se les pregunta y se apuntan en la pizarra, se les pide que las devuelvan y se quedan cerca del mago. Este hace un gesto mágico y las tres cuerdas acaban teniendo la misma longitud. Tras enseñarlas, el mago pide a cada alumno que agarre los extremos de cada cuerda y se les pregunta cuánto medían antes. A la cuenta de tres el mago suelta las cuerdas y cada alumno tiene una cuerda con un tamaño distinto, recibiendo el aplauso de los compañeros. En la Figura 3 se muestran las imágenes de este efecto.

\subsection{Contemos hasta diez (De Vecchi, 2009)}

Contenidos: números del uno al diez en español, inglés, gallego y finés.

Competencias: competencia en comunicación lingüística, conciencia y expresiones culturales, competencia matemática y competencias básicas en ciencia y tecnología.

\section{Figura 4}
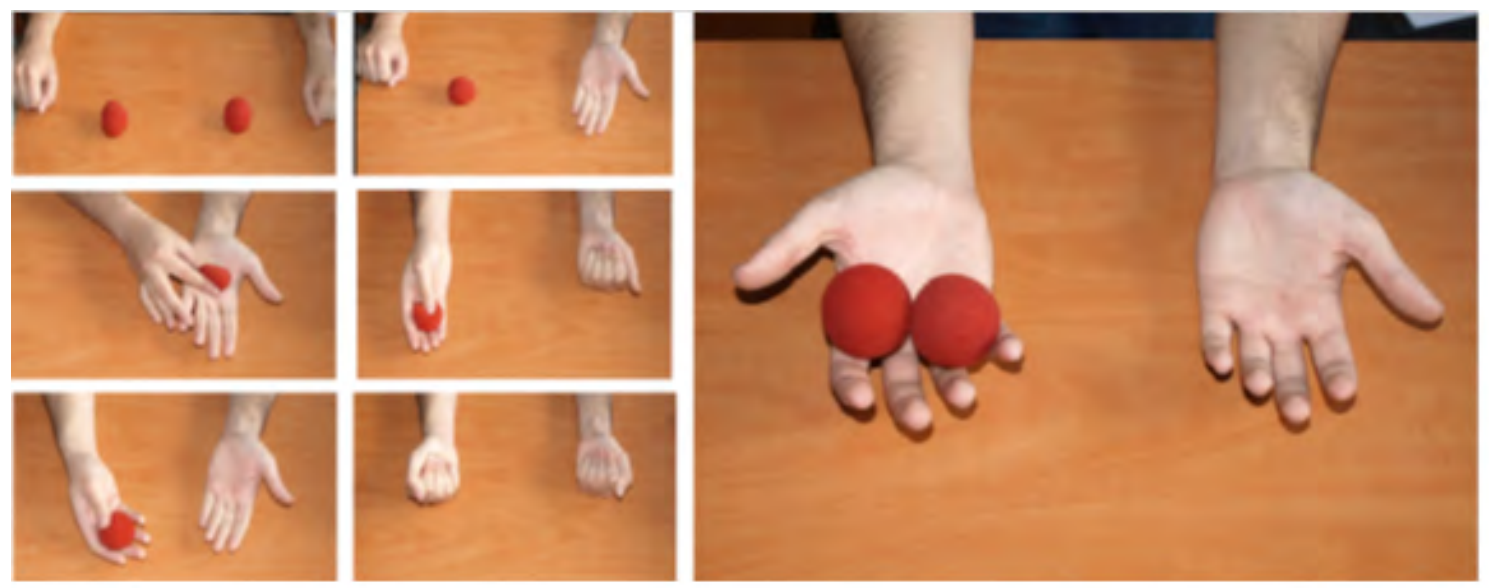

Efecto: El mago pide a un alumno que sea su asistente. Le da a examinar dos bolas de esponja y le pide que ponga las manos palma arriba, usando al alumno no solo como mesa para apoyar las esponjas, sino que este tendrá una visión diferente al resto de sus compañeros. Para realizar el juego el mago debe realizar un rito y decir todos juntos unas palabras mágicas, en este caso los números del uno al diez. Mientras los estudiantes dicen los números, el mago se pone una bola en cada mano; cuando dicen diez la bola de la mano izquierda ha desaparecido y se ha 
reunido con la de la derecha. Esto se repite dos veces más, solo que se habla en gallego y en inglés. En la última el mago dice que lo hagan en finés; como los alumnos no lo saben, el mago hace otra cosa, pone una bola en su mano y otra en el alumno que ha usado de mesa, él va diciendo los números en finés y los alumnos lo dicen. Al final el alumno tiene dos pelotas en la mano y el mago ninguna, prueba de que han hecho bien el rito. En la Figura 4 se muestran las imágenes.

\subsection{El espectador mago (Giobbi, 2004)}

Contenidos: técnicas de oratoria.

Competencias: competencia en comunicación lingüística, competencias sociales y cívicas, conciencia y expresiones culturales.

Efecto: el mago solicita ayuda a un alumno y le dice que hoy será el mago; se le pregunta por su nombre de mago; cuando el alumno lo dice, el mago lo presenta y pide un aplauso del público; el mago le dice al alumno que debe decir lo que le diga él en la oreja. En este caso el alumno coge la baraja, la mezcla y le pide al mago (que va a ser el espectador) que elija una carta, cosa que hace. Este la guarda y le toca al alumno saber cuál es. Tras un proceso de repartir las cartas en dos montones, este mira dos cartas que le indican el palo y el número de la carta que ha sido escogida. El mago-espectador saca la carta del bolsillo y es la que dijo el alumno, recibiendo el aplauso del resto de la clase.

\subsection{Predicción del dado (Gardner, 1992)}

Contenidos: característica de los dados, resolución de problemas basados en lógica.

Competencias: competencia en comunicación lingüística, competencia matemática y competencias básicas en ciencia y tecnología, sentido de la iniciativa y espíritu emprendedor.

\section{Figura 5}

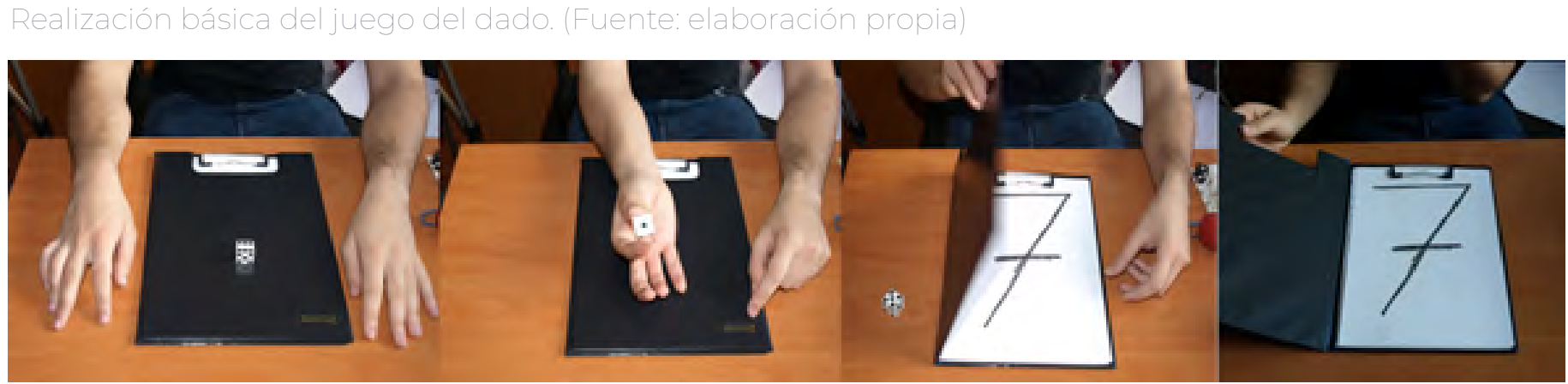

Efecto: el mago le plantea a la clase un reto, él va a hacer un juego y luego ellos deben descubrir el secreto. El mago saca a un voluntario y le da un cubilete y un dado a examinar, mientras se examinan los objetos deja un sobre en la pizarra donde lo ven todos. Se va a elegir un número de una manera aleatoria, se va a meter dentro del cubilete 
el dado, se va a agitar y lanzar a la mano del mago, el alumno recuerda el número y además suma el número del otro extremo. Cuando tenga ese número se le pregunta cuál es. El espectador dirá siete, número que se encuentra dentro de la predicción. En la Figura 5 se visualizan las imágenes de este efecto.

\subsection{Papel Okito (De Vecchi, 2009)}

Contenidos: diferentes tipos de contaminación y maneras de reducirla.

Competencias: competencia en comunicación lingüística, competencias sociales y cívicas, conciencia y expresiones culturales.

Efecto: se enseña un dibujo del mundo, el mago les pregunta a los alumnos que se fijen en el mundo, el lugar donde viven, pero el mundo está amenazado por nuestras acciones, se les pregunta cómo lo estamos dañando. Con cada cosa que van diciendo los alumnos se va rompiendo el dibujo. Cuando el dibujo ya esté roto se les pregunta cómo pueden reducir la contaminación. Cuando estos respondan se realizará un soplido mágico y se mostrará el dibujo recompuesto. En la Figura 6 se muestra la secuenciación de este juego.

\section{Figura 6}

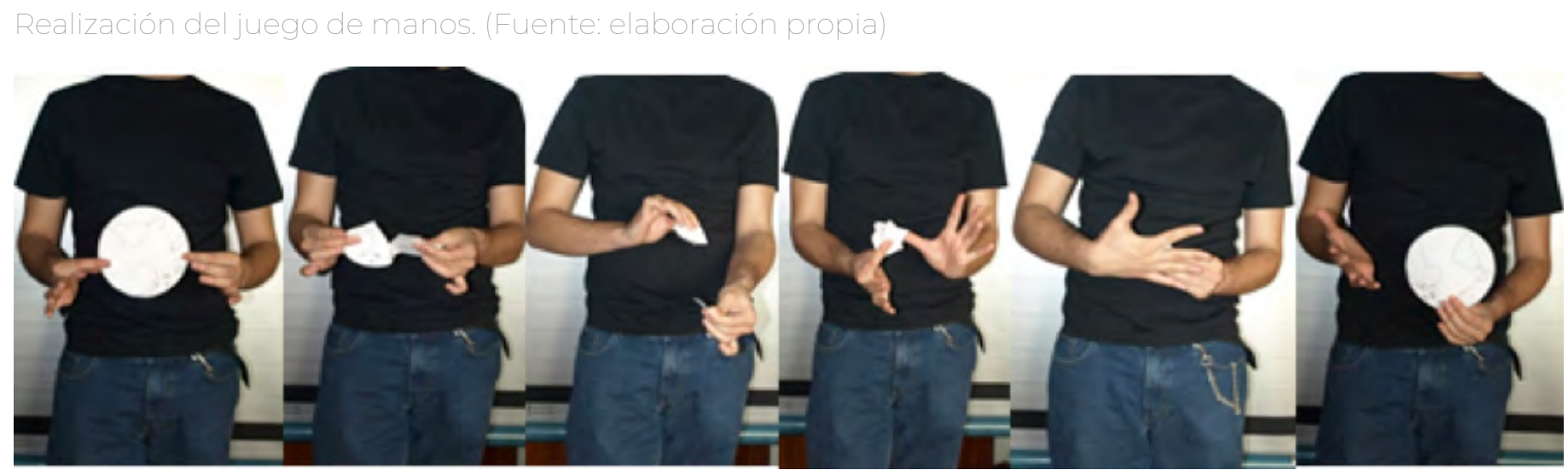

\subsection{Billete mágico (Lovick, 2006)}

Contenidos: magnitudes del dinero, concretamente los diferentes tipos de billetes de euro que existen.

Competencias: competencia en comunicación lingüística, competencia matemática y competencias básicas en ciencia y tecnología.

Efecto: se les enseña a los alumnos un billete de cinco euros, se les explica que ese es el billete de menor valor que tenemos en España. Mientras se dobla el billete se les pregunta cuál es el siguiente billete que existe, dirán el de diez, cuando se despliega el billete se ha transformado en uno de diez. Mientras están reaccionando se vuelve a doblar el billete y se les preguntas cuál es el siguiente, dirán el de veinte, se despliega el billete y se ha transformado en uno de veinte. Para acabar se pide a un alumno que abra la mano, se le mete dentro el billete de veinte y se le pide que cierre la mano con fuerza. Se les pregunta de nuevo a los alumnos cuál es el siguiente 
billete, dirán el de cincuenta, cuando el alumno abre la mano verá que el billete de veinte se ha transformado en uno de cincuenta. En la Figura 7 se muestran las imágenes.

\section{Figura 7}

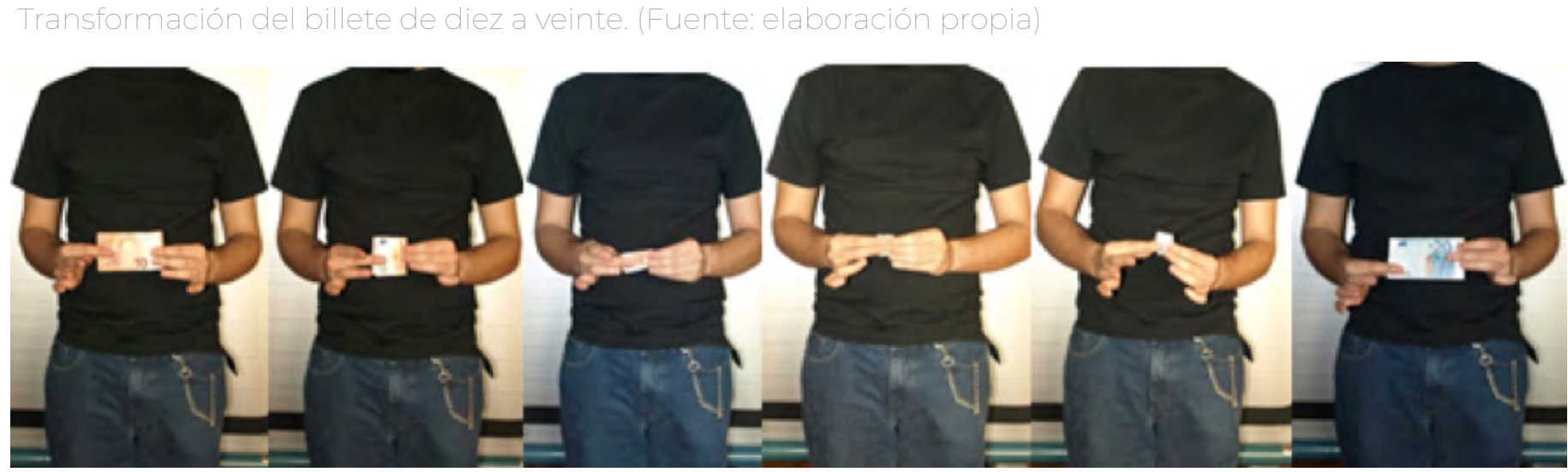

\section{EVALUACIÓN DE LA EXPERIENCIA DE AULA}

El desarrollo del proceso de enseñanza-aprendizaje debe de ser evaluado para conocer si se han alcanzado con éxito los objetivos propuestos. Para ello debemos analizar la experiencia implementada en el aula considerando tanto la actuación de los discentes como del docente.

Partiendo de que el objetivo planteado se centra en potenciar y despertar en los aprendices la motivación, el interés por participar y aprender a través del ilusionismo, esto debe manifestarse con una mejora del comportamiento y creando un clima agradable en el aula.

La evaluación de los estudiantes realizada se basó en la observación directa, con el objetivo de descubrir si estos mejoraron su comportamiento y si estaban atentos durante la realización de los juegos. A la hora de realizar las actividades, estos se mostraban participativos e ilusionados. Solo hubo algún caso particular de algún alumno que se mostraba reticente a ver la magia, pero fue solucionado cuando fue elegido como voluntario para uno de los juegos. También se pudo ver cómo mejoraron su comportamiento para poder ver la magia, pues cada vez que se les avisaba de las consecuencias de su mal comportamiento se portaban mejor. Aunque en algunos casos no se realizó el juego de magia porque llegaron a los tres "strikes", en estos casos coincidía que había algún evento externo que les excitaba como la llegada de las vacaciones.

Como las familias desempeñan un papel importante en todo proceso de aprendizaje se les formuló una encuesta para que reflexionasen y evaluasen si esta mejora del comportamiento también fue apreciada por los progenitores. En la Tabla 1, se puede apreciar lo indicado en una de dichas encuestas. 
Tabla 1

Indicación de un padre sobre la actividad y su uso como refuerzo positivo. Se ha sustituido el nombre del alumno por XXX. (Fuente: elaboración propia).

INDIQUE AQUí ALGUNA SUGERENCIA QUE PUEDA MEJORAR LA EXPERIENCIA EDUCATIVA

Por parte de XXX ha sido una experiencia muy positiva y me parece una forma innovadora y muy creativa de incentivar la imaginación, la lógica y el pensamiento abstracto.

Incluso, como refuerzo positivo para el buen comportamiento en el aula fue muy productivo.

XXX estaba siempre preocupado porque si se portaba mal, conseguían tres strikes y no había magia, y eso para él era un castigo importante.

Creo que ha sido una actividad muy buena y educativa.

Es importante que el docente realice una autoevaluación. Para ello, se utilizó un análisis DAFO de este proyecto. En el análisis interno se incluyen las debilidades, es decir aquellos recursos y situaciones que limitan el aprovechamiento de las oportunidades, y las fortalezas que son las capacidades, potenciales y elementos fuertes beneficiosos para el posicionamiento y el progreso. En el análisis externo se incluyen las amenazas o aquellas fuerzas contraproducentes que limitan el progreso del proyecto y las oportunidades o situaciones positivas que el entorno genera y que deben aprovecharse para el desarrollo del proyecto. Los resultados obtenidos se muestran en la Tabla 2.

\section{Tabla 2}

Análisis DAFO (Fuente: Elaboración propia)

\section{DEBILIDADES} AMENAZAS

Hacer un juego de magia que no presenta ningún contenido Posible sentimiento de que la magia no puede enseñar. educativo.

Perder el respeto como docente y convertirse en un "en-

Es necesaria una mejor preparación de la actividad, dejan- tretenimiento".

do poco espacio para la improvisación.

Es difícil encontrar un juego de magia para reforzar algunos conceptos.

\section{VENTAJAS}

Los alumnos estaban motivados.

Había interés por la actividad y participación.

Se conseguía controlar el mal comportamiento.

\section{OPORTUNIDADES}

Posible participación de las familias.

Usarla para ayudar a crear un mejor ambiente en el aula. 


\section{CONCLUSIONES}

Para terminar, una vez realizada la evaluación (observación de los alumnos, encuesta a las familias y autoevaluación del docente mediante el análisis DAFO) y teniendo en cuenta sus resultados, podemos concluir como Godoy (2005) que la magia es una herramienta pedagógica que permite incrementar la concentración de los discentes, aumentar la habilidad motriz, favorecer la autoestima, desarrollar la improvisación, elevar la autoconfianza, desarrollar la creatividad y potenciar la personalidad y la comunicación.

De este modo a partir de los resultados se puede elaborar un Plan de Actuación o Mejora (Escudero, 2004). El alumno debe tener un alto nivel de motivación e interés durante todo el proceso y sentirse importante dentro del aula. Para ello y con el fin de conseguir un aprendizaje significativo con el uso de la magia, vemos necesario tener en cuenta ciertos aspectos que señala Grosso (2014): los contenidos deben ser transmitidos de forma positiva y estimulante, el docente es el encargado de transmitir el interés y curiosidad por aprender, por lo tanto, debe tener gran capacidad de comunicación y de transmisión de emociones para despertar la motivación y el deseo de aprender y subir la autoestima. Rawson (1978) manifiesta que "el mago es un narrador y las maravillas que relata parecen suceder. Así los niños se sienten fascinados por la magia. Si usted puede hacer un truco mágico, podrá disponer de la atención instantánea de cualquier niño” (p. 13).

Es importante también la familia ya que juega un papel clave dentro de este proceso. Para conseguir su apoyo y motivación el docente debe aportarle información sobre este método de trabajo y deben conocer cuáles son los objetivos planteados con esta experiencia.

Al finalizar el trabajo, somos conscientes de que se han trabajado las competencias clave, tal y como se ha indicado en cada una de las actividades y se han alcanzado los objetivos propuestos.

\section{REFERENCIAS BIBLIOGRÁFICAS}

Almau, A. (2013). Se puede enseñar Matemáticas o Física con trucos de magia. Cuadernos de Pedagogía, 433, $38-43$.

Blasco, F. (2016). Matemagia. Madrid: Arid.

Conde, A. (2013). La magia como herramienta pedagógica. Recuperado de http://alvarocondemago.blogspot.com. es/2013/09/la-magia-como-herramienta-pedagogica.html

De Vecchi (2009). Gran curso de Magia y Prestidigitación. Barcelona: De Vecchi.

Escudero, J. (2004). Análisis de la realidad local. Técnicas y métodos de investigación desde la Animación Sociocultural. Madrid: Narcea.

Etcheverry, J. (2000). La magia de Ascanio. La concepción estructural de la magia. Su pensamiento teórico-mágico. Madrid: Laura Avilés.

Fernandez, R. y Serrano, F. (2015). Matemagia y su influencia en la actitud hacia las matemáticas en la escuela rural. Números, Revista de Didáctica de las Matemáticas, 89, 33-53. 
Francisco, C., Martínez M. y Sánchez, A. (2013). Escuela de magia: competencias desde la ilusión y la fantasía en movimiento. Recuperado de http://es.calameo.com/books/004247927d444e89a8d2b

Frith, G. H. y Walker, J. C. (1983). Magic as Motivation for Handicapped Students. Teaching Exceptional Children, 15(2), 108-110.

García, E. (2011). La magia para aprender mejor. Recuperado de http://www.hoy.es/v/20110531/sociedad/magia-para-aprender-mejor-20110531.html

Gardner, M. (1992). Mathematical Circus. Washington: The Mathematical Association of America.

Giobbi, R. (2004). Roberto Extralight: juego de manos con cartas. Madrid: Páginas Libros de Magia.

Godoy, J. A. (2005). Beneficios de la magia. Recuperado de https://cancinelli.jimdo.com/beneficios-de-hacer-magial

Gómez, M. y Camañero, A. (2009). La ciencia de la magia. Alambique, 60, 24-32.

González, S. (2011). Magia didáctica. Paiderex. Revista Extremeña sobre formación y Educación, 2(4), 8-10. Recuperado de: http://revista.academiamaestre.es/2011/08/magia-didactica/

Grosso, E. (2014). Disfrutar aprendiendo. Recuperado de http://espectacularkids.com/blog/es/disfrutaraprendiendo/\#more-4705

Kaye, D. (2007). Serio de remate: cómo hacer magia para niños. Madrid: Páginas.

Koirala, H. (2005). The effect of mathmagic on the algebraic knowledge and skills of low-performing high school students. En H. L. Chick y J. L. Vincent (Eds.), Proceedings of the Twenty Ninth Annual Conference of the International Group for the Psychology of Mathematics Education (pp. 209-216). Melbourne: University of Melbourne.

Koirala, H. P. y Goodwin, P. M. (2000). Teaching algebra in the middle grades using mathmagic. Mathematics Teaching in the Middle School, 5(9), 562-566.

Lane, K. (2010). Derren Brown: Magician or mathemagician? Equals, 16(2), 14-16.

Ley Orgánica 8/2013, de 9 de diciembre, para la mejora de la calidad educativa. Boletín Oficial del Estado. Madrid, 10 de diciembre de 2013, núm. 295, pp. 97858-97921.

López, J. (2014). Using playing cards to differentiate probability interpretations. Teach. Stat., 36(3), 76-78.

Lovitt, C. y Clarke, D. (1988). The Mathematics Curriculum and Teaching Program professional development package. Canberra: Curriculum Development Centre.

Lovick, J. (2006). Switch: unfolding the $\$ 100$ bill change. Rancho Cordova: Murphy’s Magic Supplies.

Ludueña, F. (2015). El cerebro mágico. Cómo los grandes magos potencian el pensamiento y la creatividad. Madrid: Aguilar.

Martín, S. (2011). Por arte de magia. Aula del pedagogo, 4, 15-28. Recuperado de: http://www.auladelpedagogo. com/wp-content/pdf/04.pdf 
McCormack, A. J. (1985). Teaching with magic: Easy ways to hook your class on science. Learning, 14(1), 62-67.

McShea, B., Vogel, J. y Yarnevich, M. (2005). Harry Potter and the magic of mathematics. Mathematics Teaching in the Middle School, 10, 408-414.

Montesillos, M. (2014). Utilizo trucos de magia para darle un aspecto lúdico a las matemáticas. La Verdad. Recuperado de: http://www.laverdad.es/murcia/v/20140202/region/utilizo-trucos-magia-para-20140202. html

Navas, J. (2012). Juegos matemáticos, un poco de matemagia. Pensamiento matemático, 2, 209-216.

Nishiyama, Y. (2012). Increasing and decreasing of areas. Int. J. Pure Appl. Math., 80(3), 385-393.

Presto, F. (1999). Magia para niños. Madrid: Susaeta.

Rawson, C. (1978). El Gran Merlini. Como divertir a los niños con magia que usted puede hacer. México: Editorial Diana.

Real Academia Española. (2016). Diccionario de la lengua española (23a ed.). Recuperado de: http://dle.rae.es/

Ruiz, X. (2013). Educando con Magia: el ilusionismo como recurso didáctico. Madrid: Narcea.

Salovey, P, Mayer, J. D., Goldman, S. L, Turvey, C y Palfai, T. P. (1995). Emotional attention, clarity, and repair: exploring emotional intelligence using the Trait Meta-Mood Scale. En J. W. Pennebaker (Ed.), Emotion, Disclosure, ef Health (pp. 125-154). Washington: American Psychological.

Santoja, J. (2003). Ernesto y el aprendiz de matemago. Madrid: Nivola.

Spencer, K. (2001). Hocus Focus: evaluating the pedagogical implications of integrating magic tricks in classroom instructions. Recuperado de http://www.hocusfocuseducation.com/wp-content/uploads/2013/01/JISfTE.pdf

Suárez, M. T. (2010). La magia y una nueva relación pedagógica con la infancia. Praxis \& Saber. Revista de Investigación y pedagogía, 1(2), 43-66.

Tamariz, J. (1991). La increíble historia de la magia. Madrid: Ediciones del Prado.

\section{INFORMACIÓN SOBRE LOS AUTORES}

Ignacio González-Puelles.Graduado en Educación Primaria por la Escuela Universitaria CEU de Magisterio de Vigo (Universidad de Vigo) (2016). Participó en el programa Erasmus en la Universidad de Helsinki (2015). Asistió a "I Jornadas Internacionales de Literatura Infantil y Juvenil, promoción de la lectura en prácticas educativas". Presentó la comunicación: "La figura femenina en los videojuegos" en "61 Encuentro de universitarios católicos: ¿Reinventar lo humano?”. Presenció diferentes cursos, entre ellos, "La magia como estrategia didáctica" celebrado en San Sebastián en el marco de la XXXII edición de los cursos de Verano-XXV cursos europeos de la Universidad de País Vasco- Euskal Herriko Unibertsitatea.

ignacioglez1994@gmail.com 
María Sandra Fragueiro. Doctora en Química Analítica por la Universidad de Vigo (2004) y Premio Extraordinario de Doctorado (Universidad de Vigo, 2006). Actualmente ejerce como Profesora Acreditada en la Escuela Universitaria CEU de Magisterio de Vigo en el Área de Ciencias. Es Coordinadora del Trabajo Fin de Grado en dicha Escuela. Sus principales líneas de investigación son la Aplicación de nuevas metodologías en Educación Primaria e Infantil en el área de ciencias. Además participa como investigadora en el Departamento de Química Analítica y Alimentaria en la Universidad de Vigo en el proyecto del Ministerio de Economía y Competitividad, convocatoria de proyectos I+D del programa estatal de fomento de la investigación científica y técnica de excelencia: "Detección on-site de iones, complejos metálicos y nanopartículas mediante estrategias nanoanalíticas basadas en grafeno y puntos cuánticos de carbono”.

$\triangle$ sandra.fragueirobarreiro@ceu.es 